\title{
Characteristics of patients with persistent severe disability and medically unexplained neurological symptoms: a pilot study
}

\author{
J Allanson, C Bass, D T Wade
}

J Neurol Neurosurg Psychiatry 2002;73:307-309

This study audited 25 patients $(21$ female) from Oxfordshire who had been referred to either the liaison psychiatry or the neurological disability service between 1992 and 1998, reported a Barthel activities of daily living index score $<20$ or a global assessment of functioning score of $\leqslant 30$, and had no pathology to explain their neurological disability. Levels of motor impairment, disability, mood, and cognitive status were assessed using standardised scales, and all patients were assigned a psychiatric diagnosis according to the International classification of diseases, 10th revision. Of the 25 patients, 13 had a motor conversion disorder, 8 had diverse somatoform disorders, and 3 had chronic fatigue syndrome. Nine had extensive previous contact with psychiatric services and 11 had experienced physical or sexual abuse. In 6 patients cessation of repeated self harm was closely associated with the onset of wheelchair use. Seven were receiving treatment for depression. The commonest putative diagnoses were multiple sclerosis (6) and epilepsy (5). Twelve were unable to walk and 20 owned a wheelchair but only 3 had formal care packages. The mean (SD) Barthel score was 14.1 (3.3) and the mean (SD) Frenchay activity index score was 12.9 (7.5). All were unemployed and receiving a disability living allowance, and some had benefits of up to $£ 1815$ a month. This small but significant group of disabled patients had a variety of psychiatric and neurological diagnoses and used considerable health care resources.

M any patients are referred to hospital specialists with complaints that cannot be explained by underlying disease. ${ }^{1-3}$ Most patients are referred back to the primary care doctor after investigation but a proportion remain symptomatic and become significantly disabled physically. When these disabilities involve loss of or alteration in limb function patients may be referred to neurological disability services. ${ }^{4}$ The problem is of some economic importance to primary ${ }^{5}$ and secondary ${ }^{6}$ health care services. We audited patients attending services with significant "physical disability" to characterise them and to estimate a minimum prevalence of this phenomenon.

\section{METHODS}

Patients were identified from records in the liaison psychiatry department and neurological disability services in Oxford. All patients attending the liaison psychiatry outpatient clinic are given a rating of functional impairment using the global assessment of functioning (GAF) scale. ${ }^{7}$

Patients were initially contacted if they had a GAF score $\leqslant 30$ ("unable to function in many areas") or a Barthel activities of daily living (ADL) index score ${ }^{8}$ of $<20 / 20$. They were included if the Barthel score was $\leqslant 19 / 20$ and they had no identifiable or relevant organic disease to explain the disability after appropriate clinical assessment.

Each patient was interviewed at home. The Barthel ADL index score ${ }^{8}(0-20,20$ being fully independent) and Frenchay activities index score $(0-45$, where 45 indicates full involvement in domestic tasks, social events, hobbies, and employment) were recorded as measures of dependence and social participation.

All patients were given a brief neurological examination in which any abnormal movements and any major differences in ability before and during formal testing of strength were noted, and the motricity index was recorded as a measure of limb motor impairment ( $100 \%$ for full power).$^{10}$ The nine hole peg test ${ }^{11}$ was then performed and a $10 \mathrm{~m}$ walk time recorded (with one turn) if possible. Lastly, patients completed the short orientation memory and concentration test (SOMCT) ${ }^{12}$ and Beck depression inventory. ${ }^{13}$

\section{RESULTS}

Twenty five patients ( 21 female) were identified, most from the liaison psychiatry database. The mean (SD) age was 42.4 (12.4) years, and 24 were white. The average (SD) time from onset of symptoms to the assessment was 12.6 (9.5) years and the average duration of their current level of disability was 5.1 (3.7) years.

\section{Symptoms and investigations}

Subjects reported many neurological symptoms (table 1). Fourteen complained of weakness or uselessness of at least one limb, and five reported abnormal movements as a primary complaint. Ten had a persistently abnormal posture of at least one limb, and 21 described some change in sensation or pain in at least one limb. Seven had blackouts, interpreted by their family and some health care workers as epileptic seizures.

All patients had been extensively investigated. An average of six consultants (including at least one neurologist for every patient) had been involved in the assessment of each patient's complaints.

Despite documented previous consultations where the lack of any satisfactory medical explanation for the symptoms had been explained to the patient, six patients expressed the belief that they had multiple sclerosis and five that they had epilepsy.

\section{Impairments}

The patients had a variety of apparent impairments. During assessment of 11 patients some inconsistencies in performance were observed, both informally and during structured

Abbreviations: $A D L$, activities of daily living; GAF, global assessment of functioning; SOMCT, short orientation memory and concentration test 
Table 1 Main symptoms and signs in 25 patients with medically unexplained disability

\begin{tabular}{|c|c|c|}
\hline Patient & Main symptoms & Main signs \\
\hline 1 & Weak legs with abnormal sensation & Paraplegia and weak arm \\
\hline 2 & Weak right leg & Monoplegia and left foot inversion \\
\hline 3 & Knee pain, inability to walk, fits, & Paraplegia \\
\hline 4 & Fatigue and shaking of left leg & Flat, affectless facies \\
\hline 5 & Weak legs and hands, dysphagia, incontinence & Dysarthria, hemiplegia, variable tremor \\
\hline 6 & Fatigue, pain, weak left side & Minimal left sided weakness \\
\hline 7 & Blackouts with shaking of right arm & Mild left hand weakness \\
\hline 8 & Low back pain and sudden collapse & Variable incomplete paraplegia \\
\hline 9 & Proximal myalgia & Abnormally slow gait \\
\hline 10 & Blackouts, distal pain and numbness, difficulty using right hand & Abnormal gait, mild peripheral weakness \\
\hline 11 & Weak painful swollen lower legs, fatigue & Weak legs, bilateral fixed foot plantar flexion and inversion \\
\hline 12 & Useless left leg, inability to walk, fatigue & Immobility, left leg weakness \\
\hline 13 & Painful and rubbery left arm, blackouts with left arm shaking & Immobile left arm with flexed fingers \\
\hline 14 & Fatigue, myalgia & None \\
\hline 15 & Neck and arm pain with dizziness and shaking all over & Mild titubation and leg tremor, mild distal weakness \\
\hline 16 & Right sided shaking, incontinence & Immobile right lower leg, hand tremor \\
\hline 17 & Inturned left foot, left foot shaking & Plantar flexion and inversion of left foot \\
\hline 18 & Blackouts and fatigue & None \\
\hline 19 & Left sided weakness, abnormal speech, fits & Dysphasia, left hemiplegia, complete left sensory loss \\
\hline 20 & Weak legs with low back pain, previous fits & Incomplete paraplegia \\
\hline 21 & Inability to stand up because of dizziness, sensory change below waist & None \\
\hline 22 & Myalgia and fatigue & Major generalised muscle wasting \\
\hline 23 & Right leg and back pain with fatigue & None \\
\hline 24 & Low back pain, inability to stand for over 15 minutes, fatigue, fits & None \\
\hline 25 & Weak numb legs, reduced balance, impaired vision & Profound paraplegia, arm weakness \\
\hline
\end{tabular}

tasks, suggesting possible elaboration; the best scores achieved were recorded. Ten maintained an abnormal posture of at least one limb throughout the assessment (one to two hours). Three had abnormal speech. Twelve said that they were unable to walk and declined timing of a $10 \mathrm{~m}$ walk. The motricity index was $<50 \%$ on the left in four but in none on the right, and nine had bilaterally reduced scores. Laterality of impairments was not related to hand preference. During the nine hole peg test, contralateral tremor improved in one person and abnormal posture recovered in another.

\section{Disability and dependence}

The mean (SD) Barthel ADL index score was 14.1 (3.3). Eight reported major day to day variation in their ability, largely as a result of variation in pain and fatigue (largest range 10 points). Three people scored less than 9/20. Of note, all 25 had regular help with bathing, with 22 having daily help. One had an indwelling catheter for persisting urgency of micturition. Eight walked only during therapy sessions.

All patients were unemployed. All relied on family and friends to perform domestic tasks. The average (SD) Frenchay activities index score was 12.9 (7.5) out of a possible 45, indicating severe limitation in social activities. There was no correlation between Barthel ADL and Frenchay activities index scores.

The help required with personal care was provided entirely by partners, parents, or other family members in almost all cases. Only three had formal care packages (with Barthel ADL scores of 4, 8, and 10-20) and in two with severe forms of chronic fatigue syndrome carers were arranged to allow respite for other family members. Significant cognitive difficulty was rare, with 24 patients scoring $>22 / 28$ on the SOMC.

\section{Psychiatric morbidity}

All except one patient had been assessed by a psychiatrist (CB) and assigned a formal psychiatric diagnosis according to criteria in the International classification of diseases, 10th revision (1992). Thirteen had a motor conversion disorder, five somatoform pain disorder, three undifferentiated somatoform disorder, three severe chronic fatigue (neurasthenia), and one profound hypochondriasis (delusional type). Ten had scores above 17 on the Beck depression inventory and seven were being treated for depression.

Nine patients had a long history of contact with psychiatric services. Nine had taken drug overdoses, six of whom had repeatedly performed acts of deliberate self harm (over five in four cases). Five of these six patients did not take further overdoses once their level of disability had stabilised, usually once the patient had become a wheelchair user.

Eleven patients (including four of the group who had taken overdoses) gave unequivocal histories of abuse (physical or sexual) either in childhood or by previous partners.

\section{Use of health care resources and welfare benefits}

A wide range of equipment was used. Twenty had a wheelchair and 13 used it all the time. A further two used a relative's wheelchair. Eight had had either stair lifts or through floor lifts installed. Most equipment had been provided by social services after assessment by community occupational therapists. The largest grant provided was $£ 18000$ for a downstairs extension with walk in shower.

The patients received some support from statutory services. Eight were admitted for respite care, five attended day centres regularly, two attended mental health centres twice weekly, and three attended young disabled units weekly.

All patients studied were receiving a disability living allowance. Ten were receiving the maximum for care and mobility (£360 a week). Some were also entitled to incapacity benefit (£66 a week) and if their partners were also unemployed patients were entitled to income support ( $£ 80.65$ a week for a couple). The maximum sum being paid to one patient was $£ 1815$ a month (disability living allowance, income support, invalidity benefit, and rent allowance).

\section{DISCUSSION}

We identified 25 patients with an extreme form of apparent neurological "physical" disability in the absence of any neurological disease. These patients were not rare (derived from a population of 560000 ) and were using considerable health and social service resources. The two characteristic features were the variability of diagnosis, both between patients and within patients, and the close involvement of families. 
There are three main limitations. Firstly, this was not a community survey, so it is difficult to estimate the true prevalence of this form of severe non-organic disability. Our patient group provides a minimum estimate of those referred to secondary care services and interviewed by a psychiatrist. Secondly, we excluded patients presenting with severe disability who had even minimal pathology detectable on investigation. Thirdly, because the results of our measures of physical impairment and disability are dependent on effort, the data are difficult to interpret. The results reported above are the best scores achieved at any moment during the assessment.

We were surprised to find that most of the care was provided by partners and family members (only three had formal care packages). Most partners were unemployed and fully occupied as carers, which needs to be considered when managing these patients. Moreover, while most patients were receiving substantial financial support and had been provided with many items of complex equipment, only five were using other services regularly.

During this study we became increasingly aware of the lack of adequate service provision for patients with profound "non-organic" disabilities. Psychiatric services generally give low priority to this group of patients ${ }^{14}$ and staff in neurology rehabilitation services may become alienated or hostile towards such patients.

We believe that the optimum management of these patients can be provided by a multidisciplinary team with personnel from neurological disability and psychiatry services, ${ }^{15}{ }^{16}$ which should make it possible to provide, at the very least, a containing therapeutic environment and, at best, an opportunity for these patients to address relevant psychological problems and improve physical function. Such a service may benefit patients and their relatives and save costly NHS resources.

\section{ACKNOWLEDGEMENTS}

We thank all the patients for their patience and cooperation.

\section{Authors' affiliations}

J Allanson, Heberden Rehabilitation Unit, Amersham Hospital,

Amersham, Buckinghamshire HP7 OJD, UK

C Bass, Department of Psychological Medicine, John Radcliffe Hospital, Oxford OX3 9DU, UK

D T Wade, Oxford Centre for Enablement, Windmill Road, Oxford OX3 7LD, UK
Competing interests: None declared

Contributions: All authors contributed to the formulation and design of the study, interpretation, and writing the paper; JA interviewed all the patients and collected and analysed the data; $C B$ is the guarantor.

Correspondence to: Dr J Allanson; allanson@doctors.org.uk

Received 14 September 2001

In revised form 13 March 2002

Accepted 22 March 2002

\section{REFERENCES}

1 Hamilton J, Campos R, Creed F. Anxiety, depression and management of medically unexplained symptoms in medical clinics. J R Coll Physicians Lond 1996;30: 18-20.

2 Carson AJ, Ringbauer B, Stone J, et al. Do medically unexplained symptoms matter? A prospective cohort study of 300 new referrals to neurology outpatient clinics. J Neurol Neurosurg Psychiatry 2000;68:207-10

3 Reid S, Wessely S, Crayford T, et al. Medically unexplained symptoms in frequent attenders of secondary health care: retrospective cohort study. BM 2001;322:767-9.

4 Davison P, Sharpe M, Wade D, et al. "Wheelchair" patients with non-organic disease: a psychological inquiry. J Psychosom Res 1999;47:93-103

5 Barsky AJ, Ettner SL, Horsky J, et al. Resource utilization of patients with hypochondriacal health anxiety and somatization. Med Care 2001;39:705-15.

6 Williams C, House A. Reducing the cost of chronic somatisation. Ir J Psychol Med 1994;11:79-82.

7 Jones SH Thornicroft G, Coffey M, et al. A brief mental health outcome scale: reliability and validity of the global assessment of functioning (GAF). Br J Psychiatry 1995; 166:654-9.

8 Wade DT, Collin C. The Barthel ADL index: a standard measure of physical disability? Int Disabil Stud 1988;10:64-7.

9 Piercy M, Carter J, Mant J, et al. Inter-rater reliability of the Frenchay activities index in patients with stroke and their carers. Clin Rehabil 2000;14:433-40.

10 Collin C, Wade DT. Assessing motor impairment after stroke: a pilot reliability study. J Neurol Neurosurg Psychiatry 1990;53:567-79.

11 Heller A, Wade DT, Wood VA, et al. Arm function after stroke: measurement and recovery over the first three months. J Neurol Neurosurg Psychiatry 1987;50:714-9.

12 Wade DT, Vergis E. The short orientation-memory-concentration test: a study of its reliability and validity. Clin Rehabil 1999;13:164-70.

13 Beck AT, Ward CH, Mendelson M, et al. An inventory for measuring depression. Arch Gen Psychiatry 1961;4:561-71.

14 Bass C, Peveler R, House A. Somatoform disorders: severe psychiatric illnesses neglected by psychiatrists. Br J Psychiatry 2001;179:1 1-4.

15 Wade DT. Rehabilitation for hysterical conversion states: a critical review and conceptual reconstruction. In: Halligan PW, Bass C, Marshall J, eds. Contemporary approaches to the study of hysteria. Clinical and therapeutic perspectives. Oxford: Oxford University Press, 2001:330-46.

16 Wade DT. Medically unexplained disability: a misnomer and an opportunity for rehabilitation. Clin Rehabil 2001;15:343-7. 